\title{
Quantum Second Virial Coefficients for Krypton -86 Gas
}

\author{
Ibtisam. F. Al-Maaitah ${ }^{1}$ \\ ${ }^{1}$ Applied Physics Department, Tafila Technical University, Tafila, Jordan \\ Correspondence: Ibtisam. F. Al-Maaitah, Applied Physics Department, Tafila Technical University, Tafila, Jordan. \\ E-mail: ibt.f@yahoo.com
}

Received: March 2, 2018

Accepted: March 14, 2018

Online Published: April 7, 2018

doi:10.5539/apr.v10n3p1

URL: https://doi.org/10.5539/apr.v10n3p1

\begin{abstract}
A quantum second virid coefficient for krypton gas is calculated using ab initio potential, Barker et al potential and Morse potential. Calculations have been made using Galitskii-Migdal-Feynman (GMF) formalism. Agreements with experiments is good though not perfect.
\end{abstract}

Keywords: Quantum second virial coeffitient, krypton, scattering length

\section{Introduction}

Virial coefficients $B_{i} \quad$ B appear as coefficients in the virial expansion of the pressure of a many-particle system - The many-body problem is a general name for a vast category of physical problems pertaining to the properties of microscopic systems made of a large number of interacting particles- in powers of the density, providing systematic corrections to the ideal gas law. They are characteristic of the interaction potential between the particles and in general depend on the temperature. The second virial coefficient $B_{2} \quad \mathrm{~B}$ depends only on the pair interaction between the particles. Many methods have been developed for calculating second virial coefficients for $\mathrm{Kr}$ gas: A differential method is described for measuring the second virial coefficient of a gas from approximately the normal boiling-point of the substance to room temperature, given the value of the virial coefficient at one temperature (Byrne et al.,1971). The experimental second virial coefficients of $\mathrm{Ar}, \mathrm{Kr}$, and $\mathrm{Ar}-\mathrm{Kr}$ mixtures were determined for the temperature range $80-140 \mathrm{~K}$. These were $5-15 \%$ larger in magnitude than those calculated with the LennardJones 12-6 potential (Fender \& Halsey, 1962). In our work quantum second virial coefficient at temperatures above $120^{\circ} \mathrm{K}$ is calculated by using theoretical method at low density.

\section{Theoretical Framework}

\subsection{GMF T-matrix}

The starting point in computing $\mathrm{B}_{\mathrm{q}}$, then, is the determination of $\delta_{\ell}^{E}$. This can be accomplished by solving the GMF integral equation for the T-matrix, using a matrix-inversion technique. The form the T-matrix is given by (Ghassib et al., 1976):

$$
\begin{aligned}
& T_{\ell}\left(\vec{p}, \vec{p}^{\prime} ; s, \vec{P}, \beta\right)=u_{\ell}\left(\left|\vec{p}-\vec{p}^{\prime}\right|\right)-(2 \pi)^{-2} \int_{0}^{\infty} k^{2} d \vec{k} u_{\ell}(|\vec{p}-\vec{k}|) \\
\times & {\left[g_{0}(k, s) Q(\vec{k}, \vec{P}, \beta)-g_{0}^{+}(k, s) \bar{Q}(\vec{k}, \vec{P}, \beta)\right] T_{\ell}\left(\vec{k}, \vec{p}^{\prime} ; s, \vec{P}, \beta\right) }
\end{aligned}
$$

Here: $p$ and $p^{\prime}$ are the relative incoming and outgoing momenta; $\mathrm{P}$ is the center-of-mass momentum. The operator $u \equiv \frac{2 m_{r} V}{\hbar^{2}} \equiv \frac{1}{2} V$ [in natural units], $m_{r}$ being the reduced mass of the Kr interacting pair; $m_{r}=\frac{1}{2} m$, and $\mathrm{V}$ the Fourier transform of a static central two-body potential. The free two-body Green's function $\mathrm{g}_{\mathrm{o}}(\mathrm{s})$ is specified by $g_{0}(\vec{k}, s) \equiv \frac{1}{k^{2}-s-i \eta}, \eta$ being a positive infinitesimal in the scattering region ( $\left.\mathrm{s}>0\right)$ and zero otherwise, and the parameter $s$ the total energy of the interacting pair in the center-of-mass frame, given by $s \equiv 2 \mu\left(2 P_{o}-\frac{P^{2}}{m}\right)$ 
$\mathrm{P}_{0}$ is the total energy of the pair and $\mathrm{P}^{2}$ is the energy carried by the center of mass.

For a many-bosonic system, the operator $Q(\bar{Q})$ is the product of two occupation probabilities as follows (Joudeh et al., 2010):

$Q(k, P, \beta)=(1+n(|\vec{k}-\vec{P}|))(1+n(|\vec{k}+\vec{P}|)), \bar{Q}(k, P, \beta)=n(|\vec{k}-\vec{P}|) n(|\vec{k}+\vec{P}|)$, The parameterized $T_{\ell}(p ; P ; \beta)$

can be expressed in terms of real many-body shifts $\delta_{\ell}^{E}(p ; P, \beta)$ according to (Bishop et al.,1976):

$$
T_{\ell}(p ; P ; \beta)=\operatorname{Im} T_{\ell}(p ; P ; \beta)+\operatorname{Re} T_{\ell}(p ; P ; \beta)
$$

Where, $\operatorname{Im} T_{\ell}(p ; P ; \beta)$ and $\operatorname{Re} T_{\ell}(p ; P ; \beta)$ denote, respectively, the imaginary and real parts of $T_{\ell}(p ; P ; \beta)$; they are defined by

$$
\begin{aligned}
& \operatorname{Re} T_{\ell}(p ; P ; \beta)=-\frac{2 \pi}{p(Q(p ; P, \beta)+\bar{Q}(p ; P, \beta))} \sin \left(2 \delta_{\ell}^{E}(p ; P, \beta)\right) \\
& \operatorname{Im} T_{\ell}(p ; P ; \beta)=-\frac{2 \pi}{p(Q(p ; P, \beta)+\bar{Q}(p ; P, \beta))}\left(1-\cos \left(2 \delta_{\ell}^{E}(p ; P, \beta)\right)\right)
\end{aligned}
$$

so that,

$$
\delta_{\ell}^{E}(p ; P, \beta) \equiv \tan ^{-1} \frac{\operatorname{Im} T_{\ell}(p ; P ; \beta)}{\operatorname{Re} T_{\ell}(p ; P ; \beta)}
$$

2.2 The Second Virial Coefficient \& the Beth-Uhlenbeck Formula

The Beth-Uhlenbeck formula gives a quantum expression of second virial coefficient $\mathrm{B}_{\mathrm{q}}(\mathrm{T})$ (Seguin et al., 1987):

$$
B_{q}(T)=-\frac{\lambda^{3}}{2^{5 / 2}}-2^{3 / 2} \lambda^{3} \sum_{E_{B}}\left(e^{-\beta E_{B}}-1\right)-\frac{2^{3 / 2} \lambda^{5}}{\pi^{2}} \int_{0}^{\infty} d k k \sum_{\ell(\text { even })}^{\infty}(2 \ell+1) \delta_{\ell}^{E}(k) e^{-\beta E(k)}
$$

Here $\lambda=\left(8 \pi \hbar^{2} / m k_{B} T\right)^{1 / 2}$ is the thermal de Broglie wavelength, $\mathrm{E}_{\mathrm{B}}$ denotes bound-state energies and $\delta_{\ell}^{E}(k)$ is

the 'effective' $\ell$-partial phase shift corresponding to energy $E(k)=\hbar^{2} k^{2} / 2 m$. The word 'effective' indicates that the quantities involved pertain to a many-body medium; they depend on the density and temperature. The first term on the right-hand side is the 'ideal Bose gas' term which diminishes with increasing temperature; it depends on statistics but not on the interaction. The second represents the contribution of bound states in the system; it is related to its discrete energy levels. However, this term can be neglected (Dardi \& Dahler, 1991). The third term represents the contribution of scattering states; it is the major term in $\mathrm{B}_{\mathrm{q}}$ (Ghassib, 2014).

\subsection{Potential Functions and Appropriate Parameters:}

1) The $\mathrm{Kr}-\mathrm{Kr}$ potential derived by Barker et al. (1974) is:

$$
\begin{gathered}
V(r)=\varepsilon V^{*}(r), V^{*}(x)=V_{O}(r)+V_{1}(r), \\
V_{0}(r)=\sum_{i=0}^{i=5} A_{i}(r-1)^{i} \exp ^{\alpha(1-r)}-\sum_{i=0}^{2} c_{2 i+6} /\left(r^{2 i+6}+\delta\right) \\
\left.V_{1}(r)=\left[P(r-1)^{4}+Q(r-1)^{5}\right] e^{-(1-r)}, r\right\rangle 1 \\
=0 \quad, r\langle 1 \\
\mathrm{r}=\mathrm{R} / \mathrm{R}_{\mathrm{m}} ; \mathrm{R}_{\mathrm{m}}=4.008 \AA ; \quad \mathrm{A}_{0}=0.23526 ; \varepsilon / \mathrm{k}(\mathrm{K})=201.3 ; \mathrm{Ro}=(\mathrm{A}) 3.580 ; \mathrm{A}_{0}=0.23526 ; \mathrm{A}_{1}=-4.78686 ; \\
\mathrm{A}_{2}=-9.2 ; \mathrm{A} 3=-20.0 ; \mathrm{A}_{4}=-60.0-30.010 .0 ; \mathrm{A}_{5}=-114.0 ; \mathrm{C}_{6}=1.0632 ; \mathrm{C}_{8}=0.1701 ; \mathrm{C}_{10}=0.0143 ; \quad \alpha \\
=12.5 ; \delta=0.01 ; \mathrm{P}=0 ; \mathrm{Q}=0 ; \alpha=0
\end{gathered}
$$


2) Ab initio potential for $\mathrm{Kr}-\mathrm{Kr}$ interaction is specified by (Nasrabad, 2008):

$$
\begin{gathered}
V(R)=A e^{\left(-\alpha R+\beta R^{2}\right)}+\sum_{n=3}^{6} c_{2 n} /(R)^{2 n} \\
\mathrm{~A}(\mathrm{~K})=4.7306612 \times 10^{7} ; \alpha=1.35832 ; \beta=-0.032347 ; \mathrm{C}_{6}(\mathrm{~K})=-4.31194 \times 10^{7} \\
\mathrm{C}_{8}(\mathrm{~K})=-8.781526 \times 10^{8} ; \mathrm{C}_{10}(\mathrm{~K})=-1.02509451011 ; \mathrm{C}_{12}(\mathrm{~K})=1.3378236 \times 10^{12}
\end{gathered}
$$

3) Morse Potential (Naturforsch,1987):

$$
V(r)=-2 D e^{\left[-\alpha\left(r-r_{e}\right)\right]}+D e^{\left[-2 \alpha\left(r-r_{e}\right)\right]}
$$

Where; $\mathrm{D} / \mathrm{k}(\mathrm{K})=149.0 ; \mathrm{r}_{\mathrm{e}}=4.49 \AA ; \alpha=1.105 \AA^{-1}$

\section{Results and Discussion}

The results of our work and previous experimental (Sengers et al., 1971) are summarized in Table 1 and Figs.1-3.

Table 1. The quantum second virial coefficient, $\mathrm{B}_{\mathrm{q}}\left(\mathrm{cm}^{3} / \mathrm{mol}\right)$, at different temperatures, $\mathrm{T}(\mathrm{K})$, at $\mathrm{n}=1 \times 10^{20}$ atoms $/ \mathrm{m}^{-3}$ with previous experimental value

\begin{tabular}{ccccc}
\hline $\mathrm{T}[\mathrm{K}]$ & $\mathrm{B}_{\mathrm{q}}$ (Morse pot.) & $\mathrm{B}_{\mathrm{q}}$ (Barker et al. pot. $)$ & $\mathrm{B}_{\mathrm{q}}$ (ab initio pot.) & $\mathrm{B}_{\text {exp. }}$ \\
\hline 120 & -304.10 & -303.10 & -303.05 & -307.8 \\
124 & -285.43 & -284.99 & -284.80 & -288.8 \\
128 & -268.36 & -268.17 & -267.91 & -271.6 \\
132 & -253.21 & -253.07 & -252.70 & -256.1 \\
136 & -239.40 & -239 & -238.81 & -242.0 \\
140 & -226.89 & -226.65 & -226.67 & -229.1 \\
144 & -215.77 & -215.59 & -215.20 & -217.3 \\
148 & -205.14 & -204.91 & -204.91 & -206.4 \\
156 & -186.59 & -186.48 & -186.12 & -187.1 \\
160 & -178.19 & -177.98 & -177.82 & -178.5 \\
164 & -170.24 & -169.99 & -169.90 & -170.5 \\
168 & -162.85 & -162.69 & -162.58 & -163.1 \\
172 & -155.85 & -155.64 & -155.52 & -156.1 \\
176 & -149.22 & -149.06 & -149.01 & -149.5 \\
180 & -143.10 & -142.97 & -142.84 & -143.4 \\
186 & -134.63 & -134.55 & -134.42 & -134.9 \\
192 & -126.86 & -126.78 & -126.70 & -127.1 \\
198 & -119.70 & -119.63 & -119.51 & -119.9 \\
\hline
\end{tabular}

Clearly, from table 1, the quantum second virial coefficient, $B_{q}(T)$, is an increasing function of temperature throughout most of the useful temperature range. (It does decrease slightly at high temperatures.). $\mathrm{B}_{\mathrm{q}}$ is predominantly negative; but it becomes less negative as $\mathrm{T}$ increases. This can be explained as follows: At low $\mathrm{T}$, the mean energies of the atoms in the gas are of the same order of magnitude as the depth of the potential well; so that the interacting atoms spend most of their time in the attractive region of the potential. This results in a decrease in the gas pressure which leads to a negative $\mathrm{B}(\mathrm{T})$. At high $\mathrm{T}$, the average energies of the atoms increase; so that the predominant contribution to $\mathrm{B}(\mathrm{T})$ arises from the repulsive portion of the potential. This causes an increase in the gas pressure and, hence, $\mathrm{B}(\mathrm{T})$ becomes less negative (Gans, 1994). 


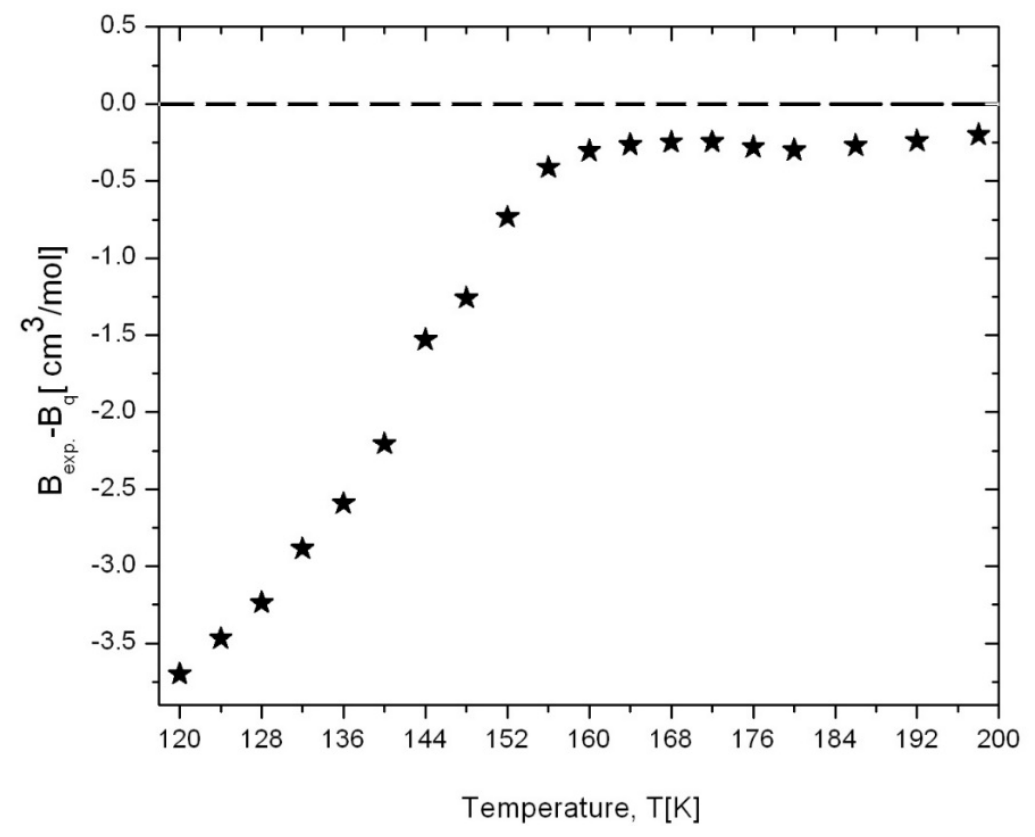

Figure 1. Deviation plot for second virial coefficients $\left[\mathrm{cm}^{3} / \mathrm{mole}\right]$ as a function of temperature $\mathrm{T}[\mathrm{K}]$ where the potential is Mores potential

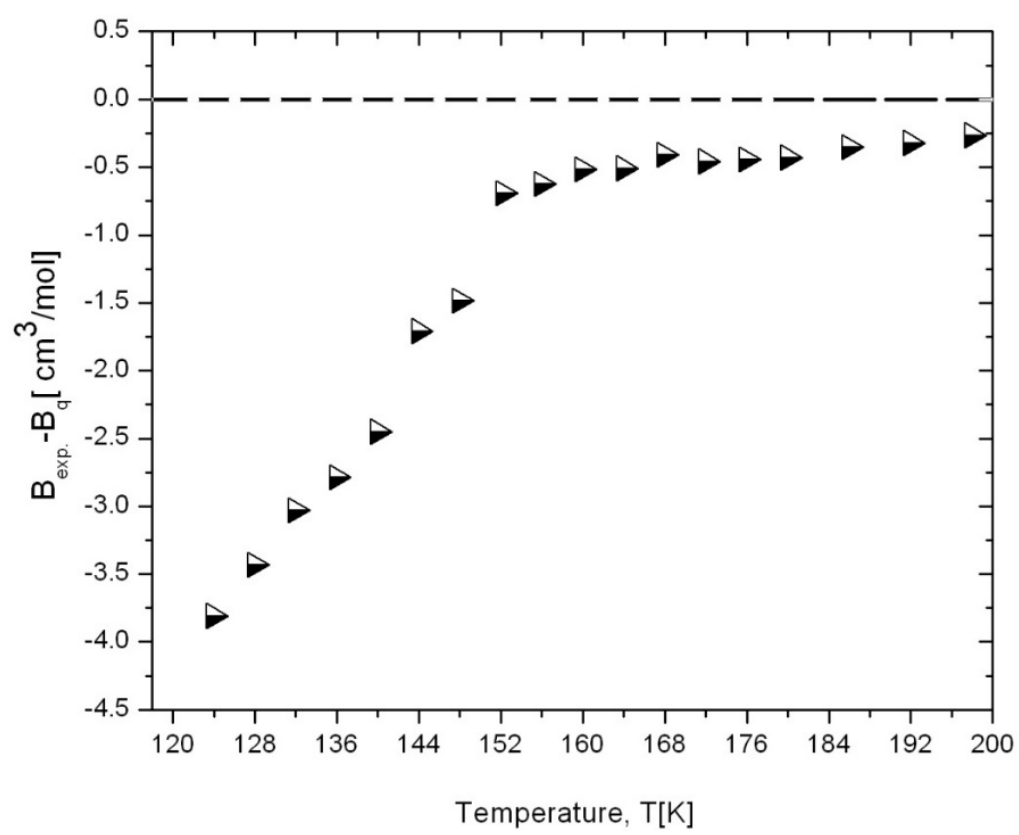

Figure 2. Deviation plot for second virial coefficients $\left[\mathrm{cm}^{3} / \mathrm{mole}\right]$ as a function of temperature $\mathrm{T}[\mathrm{K}]$ where the potential is Barker et al. potential 


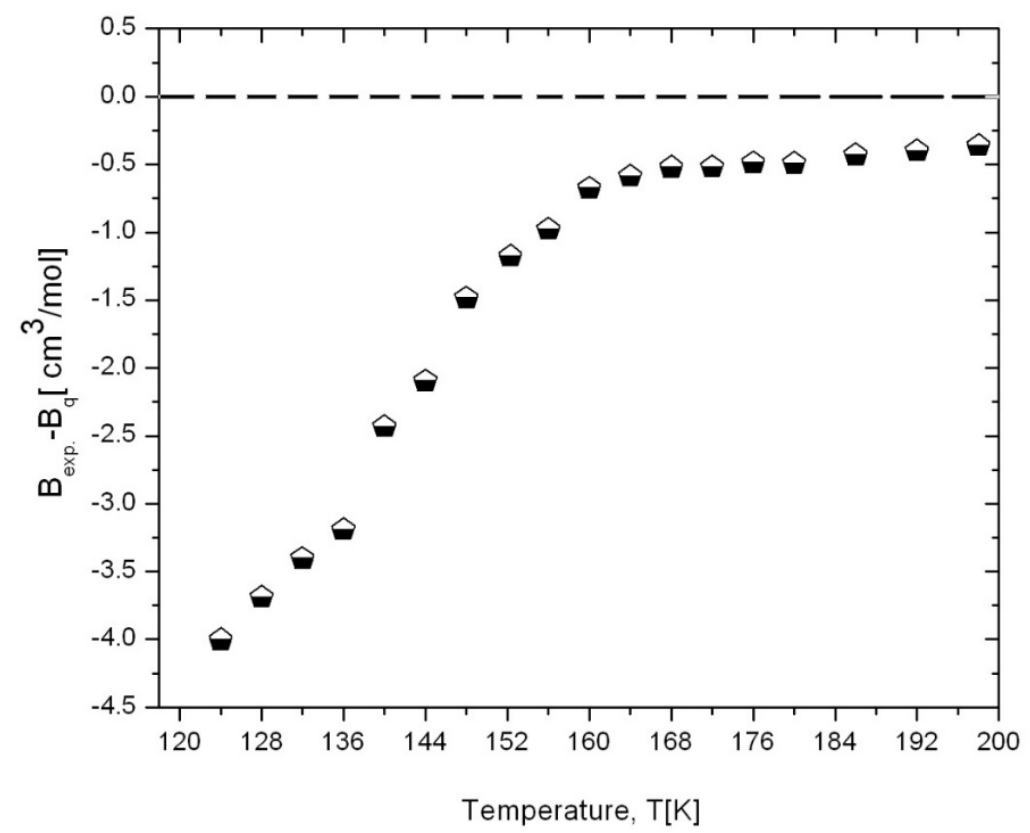

Figure 3. Deviation plot for second virial coefficients $[\mathrm{cm} 3 / \mathrm{mole}]$ as a function of temperature $\mathrm{T}[\mathrm{K}]$ where the potential is ab initio potential

From Figs. 1-3 we noted, the excellent agreement of potential Mores with that of experimental data than another potentials so the Morse potential has the advantage of enabling analytical solutions of quantum mechanical problems. In Fig. 1the second virial coefficient shows good agreement with experiment except at temperatures below $156 \mathrm{~K}$ where the experimental values become appreciably more negative than those calculated either with Mores potential, but in Fig. 2 the second virial coefficient shows good agreement with experiment except at temperatures below $152 \mathrm{~K}$. In Fig. 3 except at temperatures below $160 \mathrm{~K}$. This deviations may arise from quantum effects that are relatively small in krypton.

\section{Conclusion}

The second virial coefficient had roughly the correct behavior as it decreases monotonically when temperature is lowered. The second virial coefficients show good agreement with experimental values except at temperatures below 156 (Mores potential), 152 (Barker et al.) and 160 (ab initio potential) $\mathrm{K}$ where the experimental values become appreciably more negative than those calculated either with potentials in our work.

\section{References}

Barker, J. A., Watts, R. O., Jong, K. L., Schafer, T. P., \& Lee, Y. T. (1974). Interatomic potentials for krypton and xenon. The Journal of Chemical Physics, 61, 3081-3089.

Bishop, R. F., Ghassib, H. B., \& Strayer, M. R. (1976). Composite Pairs and Effective Two-Body Scattering in a Many-Body Medium. Physical Review A, 13, 1570-1580.

Byrne M. A., Jones, M. R., \& Staveley, L. A. K. (1971). Second virial coefficients of argon, krypton and methane and their binary mixtures at low temperatures. Transactions of the Faraday Society, 67, 1-3681.

Dardi, S. P., \& Dahler, S. J. (1991). Classical and Quantal Calculations of the Dimerization Constant and Second Virial Coefficient for Argon. Theoretica Chemica Acta, 82, 117-129.

Fender. B. E. F., \& Halsey, Jr. G. D. (1962). Second Virial Coefficients of Argon, Krypton, and ArgonKrypton Mixtures at Low Temperatures. Journal of Chemical Physics, 36, 1881-1888.

Gans, P. J. (1994). Real Gases. Physical Chemistry l, 25, 0651-0662.

Ghassib, H. B. (2014). Private Communication.

Ghassib, H. B., Bishop, R. F., \& Strayer, M. R. (1976). A Study of the Galitskii-Feynman T-Matrix for Liquid ${ }^{3}$ He. Journal of Low Temperature Physics, 23, 393-401. 
Joudeh, B. R., Sandouqa, A. S., Ghassib, H. B., \& Al-Sugheir, M. K. (2010). $3 \mathrm{He}-{ }^{3} \mathrm{He}$ and ${ }^{4} \mathrm{He}-{ }^{4} \mathrm{He}$ Cross Sections in Matter at Low Temperature. Journal of Low Temperature Physics, 161, 348-366.

Nasrabad A. E. (2008). Theory and atomistic simulation of krypton fluid. The Journal of Chemical Physics, 129, 244504.

Naturforsch, Z. (1987). Parameters of the Morse Potential from Second Virial Coefficients of Gases, Akira Matsumoto. College of Integrated Arts and Sciences, University of Osaka Prefecture, Sakai, Osaka, 591, Japan, 42a, 447-450.

Seguin, V., Guignes, H., \& Lhuillier, C. (1987). Virial Calculations for $\mathrm{H} \downarrow$ in Two and Three Dimensions. Physical Review B, 36, 141-155.

Sengers, J. M., Klein, M., \& Gallagher, J S. (1971). Pressure-Volume-Temperature Relationships of gases-Virial Coefficients (released report from Arnold Engineering Development Centre).

\section{Copyrights}

Copyright for this article is retained by the author(s), with first publication rights granted to the journal.

This is an open-access article distributed under the terms and conditions of the Creative Commons Attribution license (http://creativecommons.org/licenses/by/4.0/). 